\title{
Lost to follow-up: Challenges to conducting orthopaedic research in South Africa
}

\author{
D H S Badenhorst, ${ }^{1} \mathrm{MB}$ ChB, FC Orth (SA), MMed (Orth); C A van der Westhuizen, ${ }^{2} \mathrm{MB}$ ChB, FC Orth (SA); E Britz, ${ }^{1} \mathrm{MB}$ ChB; \\ M C Burger, ${ }^{1}$ BSc, BMedSc Hons, MMedSc, PhD; N Ferreira, ${ }^{1}$ BSc, MB ChB, FC Orth (SA), MMed (Orth), PhD \\ ${ }^{1}$ Division of Orthopaedic Surgery, Department of Surgical Sciences, Faculty of Medicine and Health Sciences, Stellenbosch University and \\ Tygerberg Hospital, Cape Town, South Africa \\ ${ }^{2}$ Division of Orthopaedic Surgery, Kimberley Provincial Hospital, Northern Cape, South Africa
}

Corresponding author: D H S Badenhorst (delmarba@gmail.com)

\begin{abstract}
Loss to follow-up poses a major problem for clinicians and researchers, and several factors that may increase its risk have been postulated. The objective of this study was to describe potential factors that contribute to loss to follow-up as seen in orthopaedic patients participating in a research study and attending the sole public orthopaedic service provider in the Northern Cape Province of South Africa (SA). All patients who underwent ankle fracture surgery at Kimberley Provincial Hospital between January 2012 and July 2013 were included, and the number of follow-up visits attended by each participant was recorded prospectively. Demographic information pertaining to travel distance, social circumstances and comorbid conditions was captured and reviewed. A total of 268 patients (male $n=112,41.8 \%$ and female $n=156,58.2 \%)$ were included. The mean (standard deviation (SD)) age was 42.3 (13.8) years (95\% confidence interval (CI) 40.6 - 43.9 , $n=266)$ and the mean body mass index (BMI, $\mathrm{kg} / \mathrm{m}^{2}$ ) was 28.0 (6.5) (95\% CI $27.2-28.8, n=251$ ), the BMI for females being 30.2 (6.1) (95\% CI 29.3 - 31.2, n=152) compared with 24.6 (5.7) (95\% CI 23.4 - 25.7, $n=99)$ for males. After excluding local patients living within $5 \mathrm{~km}$ of the hospital ( $n=77$ ), the mean travel distance was $460 \mathrm{~km}$ (range 10 - 910). There was a significant association between the number of follow-up visits attended and travel distance (incidence rate ratio (IRR) 0.999, 95\% CI $0.999-1.000 ; p=0.030$ ), BMI (IRR 0.980, $95 \%$ CI 0.966 - 0.994 ; $p=0.004$ ) and HIV status (IRR $0.841,95 \%$ CI $0.725-0.975 ; p=0.022$ ). The main factors identified in this study that influenced the number of follow-up visits attended were travel distance, BMI and HIV status. BMI was a unique finding in our study. It was identified to be a significant contributing factor to the loss to follow-up. BMI was not a contributing factor in other studies..
\end{abstract}

S Afr Med J 2018;108(11):917-921. DOI:10.7196/SAMJ.2018.v108i11.13252

Kimberley Provincial Hospital provides the sole public sector orthopaedic surgical service to the entire Northern Cape Province of South Africa (SA). Ankle fractures form part of the trauma burden and pose a challenge owing to high numbers and limited resources. The incidence of ankle fracture is reported to be $169.7 / 100$ 000/year. ${ }^{[1]}$ Currently there are no statistics on the incidence in the Northern Cape. An alternative surgical method of treatment was explored in the form of a prospective cohort series, to increase turnaround time of patients needing surgery and thus improve service delivery. Data collection while conducting this prospective trial highlighted loss to follow-up in ankle fracture patients, which prompted this report.

Numerous studies have highlighted the challenges in terms of loss to follow-up when conducting trials in musculoskeletal injuries. ${ }^{[2-5]}$ The main factors contributing to this loss to follow-up are reported to be socioeconomic, and include level of education, poverty, male gender, smoking and alcohol abuse. ${ }^{[6]}$

Young individuals as well as the very elderly are prone to be lost to follow-up. Potential reasons for this vary, but are hypothesised to include an increased frequency of substance abuse in younger populations and lack of mobility in older populations. ${ }^{[2,7]}$ In addition, smokers are reported to have an $80 \%$ higher risk of loss to follow-up compared with non-smokers. The reason for this is not clear, but it has been postulated that individuals with substance use may lack motivation to change their behaviour for health-related purposes. ${ }^{[2]}$ Several other studies also report smokers to be at risk of not attending for follow-up as expected. ${ }^{[4,5,8]}$
A recent study reported that $46.3 \%$ of all patients who failed to follow up reported 'feeling good' and hence did not feel the need to follow up, subsequently failing to report for scheduled follow-up visits. ${ }^{[3]}$ When these reported factors are combined with long travel distances and poverty, the likelihood of return is further decreased. ${ }^{[3]}$

Lack of follow-up can often lead to skewed results and influence study outcomes and validity. ${ }^{[3,4,9,10]}$ This further creates the perception of poor study design, management and execution or poor performance of the researchers or clinicians, with the impression that patients were dissatisfied with their care and chose to visit alternative clinicians. ${ }^{[10]}$ Although a genuine concern in private settings both locally and abroad, the possibility of a patient utilising the public healthcare system in SA failing to report for follow-up because of dissatisfaction with a primary healthcare provider is unlikely, because of the setup of the public healthcare system.

The Northern Cape is the largest and least densely populated province in SA. Its land area of $372889 \mathrm{~km}^{2}$ and (according to 2011 statistics $\left.{ }^{[11]}\right)$ its population of 1.145 million translates to a population density of 3.1 per $\mathrm{km}^{2}$. Placing the Northern Cape size in context, the land area of the UK fits into SA eight times, and into the Northern Cape twice (Fig. 1, A and B).

The Northern Cape has high unemployment numbers, with Stats SA reporting in 2011 that $24 \%$ of the 1.145 million people living in the province were unemployed. ${ }^{[11]}$ The average household income in 2011 was ZAR86 183 ( USD6 950) per annum, an average household consisting of between six and eight family members. Education 
levels are low, with only $60 \%$ of 18 -year-olds and $40 \%$ of 19 -year-olds attending school; only $10 \%$ of individuals aged $>19$ years attended school, and of the population group aged $>20$ years, $19.3 \%$ had no schooling, $15.8 \%$ had grade 12 education and $5.9 \%$ had a higher qualification. Furthermore, only $6 \%$ of the Northern Cape population are reported to have access to the internet. ${ }^{[11]}$

The widespread poverty in the Northern Cape means that most patients who rely on government health services also depend on the government transport system that is tasked with transporting patients across the province to their required healthcare facilities. A recent study in the USA reported that travel distance was not related to loss to follow-up, ${ }^{[4]}$ but in the developing world setting of the Northern Cape, vast distances

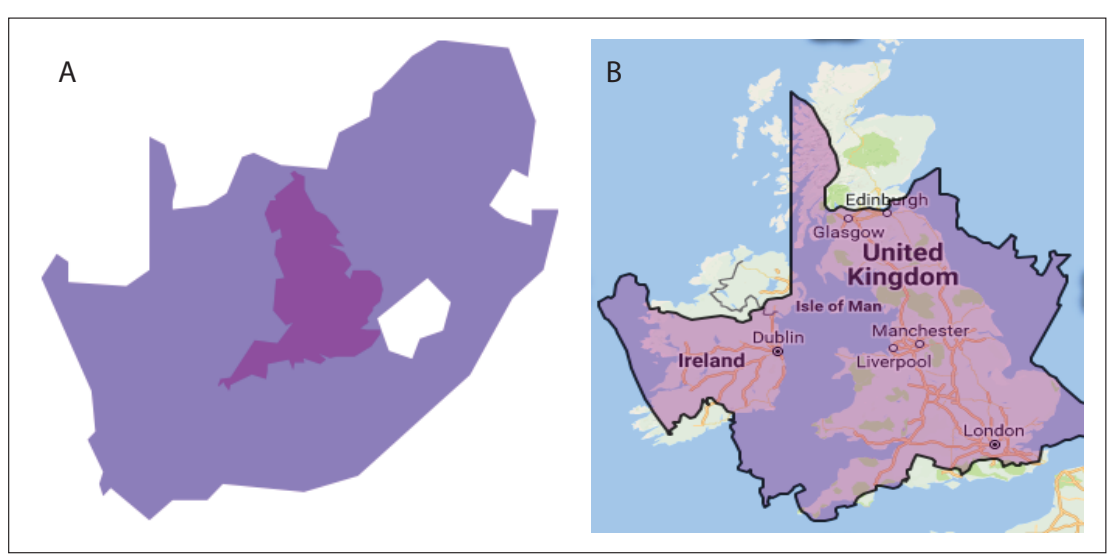

Fig. 1. (A) UK land area (purple) compared with South Africa (blue), and (B) Northern Cape Province size (overlay) compared with the UK.

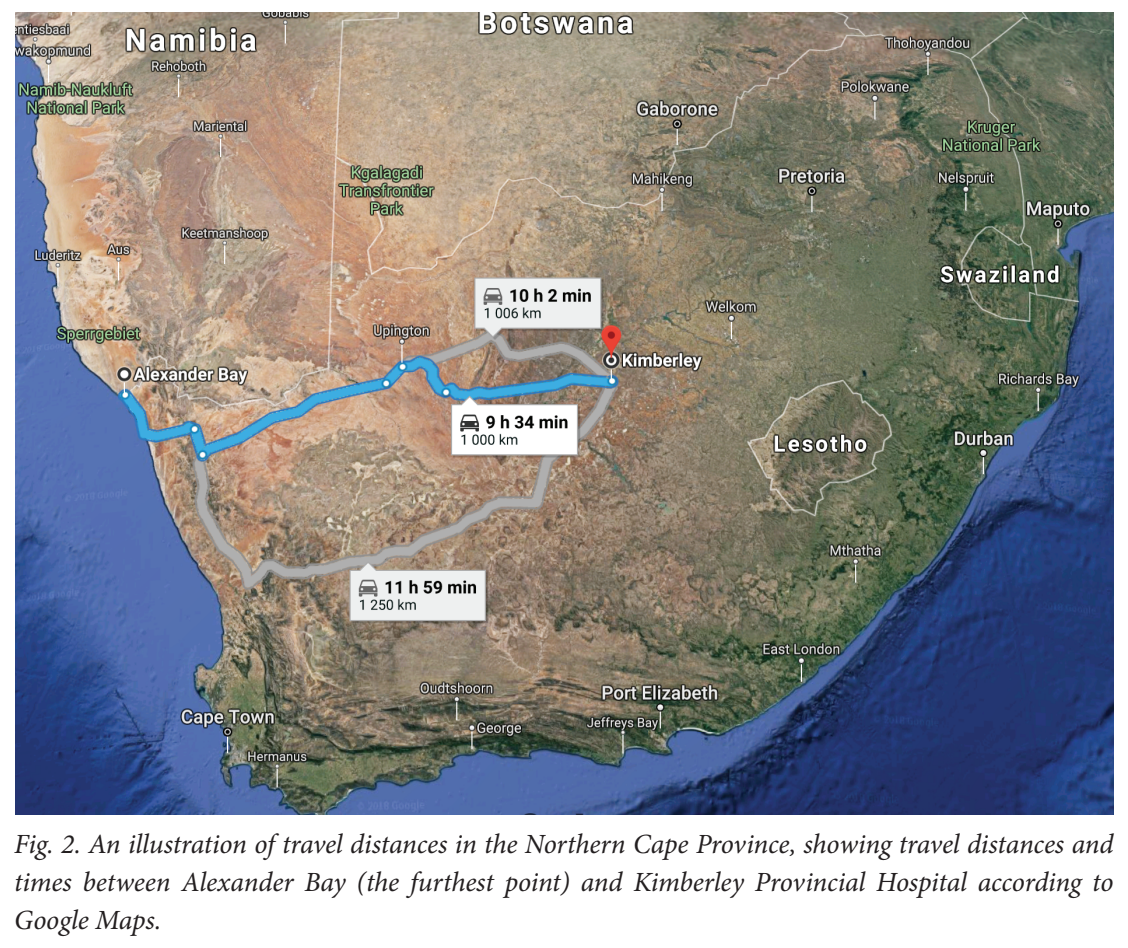

Fig. 2. An illustration of travel distances in the Northern Cape Province, showing travel distances and times between Alexander Bay (the furthest point) and Kimberley Provincial Hospital according to Google Maps.

(Fig. 2) together with poverty can potentially make returning to a hospital very challenging for patients compared with those in the developed world.

\section{Objectives}

To describe factors that contribute to loss to follow-up as seen in participants in an orthopaedic prospective descriptive trial who attended the sole public orthopaedic service provider in the Northern Cape.

\section{Methods \\ Study design}

A prospective, observational study was conducted on all patients who underwent ankle fracture surgery at Kimberley Provincial Hospital, the only institution in the Northern Cape with the means to treat orthopaedic-related trauma operatively. During this study, data on attendance for follow-up visits by each participant were collected prospectively.

\section{Patients}

Demographic information on all patients admitted for ankle fracture surgery between January 2012 and July 2013 was captured and reviewed. Patients who underwent surgical stabilisation and who were included in the prospective trial were all included in this sub-study. Demographic information was collected on admission and included age, gender, body mass index (BMI, $\left.\mathrm{kg} / \mathrm{m}^{2}\right)$, HIV status, comorbidities, substance use (including smoking, alcohol and/or other drugs), contact details including phone numbers and home address (from which travel distance was also calculated), date of injury and date of surgery.

\section{Follow-up visits}

Following surgery, included patients were discharged on day 1 and requested to return for follow-up visits at 2 weeks, 6 weeks, 12 weeks, 24 weeks and 1 year following discharge. The present study focused purely on data on: (i) whether participants attended follow-up visits; and (ii) clinical outcomes during these follow-up visits, which were prospectively collected. Although this study formed part of the normal standard of care procedures and patients were merely observed during this process, all patients were paid ZAR100 for each follow-up visit for their time and expenses. If patients did not arrive for their scheduled visit, they were contacted telephonically to arrange a new date and ask the reasons for failure to attend. Follow-up consultations were again offered to those who missed or forgot their appointments. Dates for all follow-up appointments were written on a hospital card with clear instructions of the time and location, and a separate location to the usual clinic was used to interview the patients to minimise waiting time.

This study was approved by the Health Sciences Research Ethics Committee (HSREC) of the University of the Free State (ref. no. 50/2012).

\section{Statistical analysis}

Data were analysed using Statistica version 13 (Dell, USA) and Stata version 15 (StataCorp, USA). Data were tested for normality using the Kolmogorov-Smirnov test and reported as means (standard deviations (SDs)) when normally distributed or as medians with interquartile ranges (IQRs) when not 
normally distributed. In addition, general characteristics such as age and BMI are reported as means (SDs) with the number of participants with non-missing data indicated in parentheses.

Data could either be represented as count data (i.e. the number of follow-up visits that were attended by each patient, $0-5$ ) or binary data (i.e. whether a participant followed up at all or not, yes/ no). From a clinical perspective, representing as a binary outcome would have limited meaning since a patient who only attended one follow-up visit at 2 weeks should not be compared with a patient who followed up at 2 weeks, 6 weeks, 3 months, 6 months and 12 months. In addition, some clinicians would not consider a single visit 2 weeks after surgery to be sufficient follow-up. It was therefore decided to continue with the higher level of data collected (i.e. count data) and do the subsequent appropriate analyses.

The main outcome of interest was therefore the number of followup visits that each patient attended, and patients were divided into six groups, based on their follow-up status: those who attended none of the five follow-up visits were grouped into group 0 , those who attended all follow-up visits were grouped into group 5, and those who attended only a subset of scheduled follow-up visits were grouped into groups $1-4$, based on the number of follow-up visits they attended. Considering the nature of the outcome of interest, a Poisson univariate analysis was used to determine any significant relationship between individual variables (such as sex, BMI, travel distance, smoking status and specific clinical variables) and number of follow-up visits (0 - 5) attended. The minimum level of significance to enter into a multivariate model was set at $p=0.200$. A Poisson multivariate regression was then performed to evaluate factors that were independently associated with the number of follow-up visits attended.

\section{Resullts}

\section{General characteristics}

A total of 268 patients (male $n=112,41.8 \%$ and female $n=156$, $58.2 \%)$ were included. The mean (SD) age was 42.3 (13.8) years ( $95 \%$ CI 40.6 - 43.9, $n=266)$ years and the mean BMI was 28.0 (6.5) $(95 \%$ confidence interval (CI) $27.2-28.8, n=251$ ), the BMI for females being 30.2 (6.1) (95\% CI 29.3 - 31.2, $n=152$ ) compared with 24.6 (5.7) (95\% CI 23.4 - 25.7, $n=99)$ for males.

A total of 107 patients (39.9\%) reported being smokers and 89 patients (33.2\%) reported using alcohol on a regular basis (defined as more than four drinks per week). Quantifying alcohol use was problematic owing to large differences in the type and amount described by patients, types consisting of wine, beer, spirits and homemade alcohol. Five patients (1.9\%) reported using additional substances such as snuff or marijuana.

The mean (SD) travel distance to the hospital was $164.8(192.6) \mathrm{km}$ (95\% CI 141.5 - 188.1, $n=265$ ) with the median distance being $105 \mathrm{~km}$ (IQR 5 - 262) (Fig. 3). Excluding the local patients living in Kimberley $(n=77)$ within $5 \mathrm{~km}$ of the hospital, the mean travel distance was $460 \mathrm{~km}$, ranging from 10 to $910 \mathrm{~km}$.

\section{Loss to follow-up}

Of the 268 participants, 56 (20.9\%) did not attend any follow-up visit after surgery, while only 9 participants (3.3\%) attended all follow-up visits (Fig. 4).

Age, travel distance, BMI and HIV status were all independently associated with number of follow-up visits attended (Table 1) and were therefore included in the multivariate model. Interestingly, gender and smoking, both known to be risk factors for loss to followup, did not meet the predefined significance level to be included in the model $(p<0.200)$.

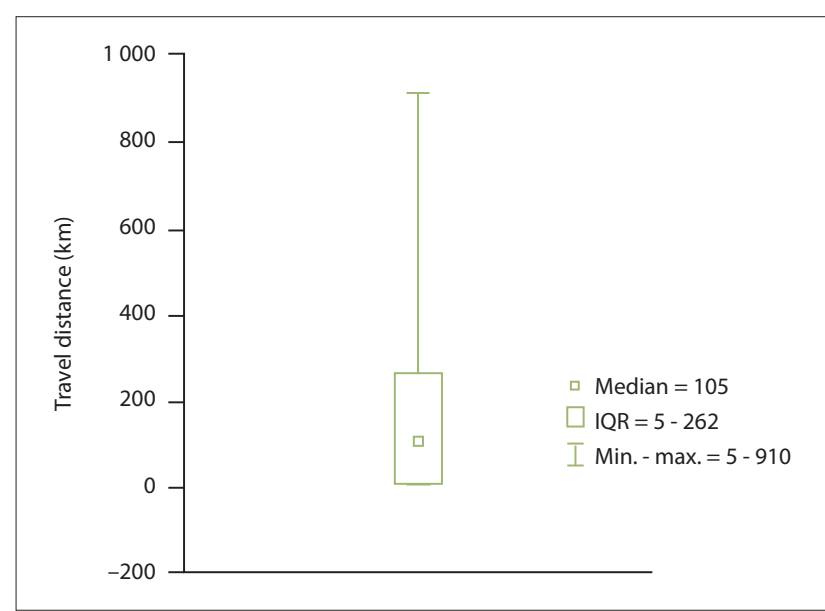

Fig. 3. Box-and-whisker plot indicating the median travel distance $(105 \mathrm{~km})$ with IQR $(5-262 \mathrm{~km})$ and minimum $/$ maximum distances (5 $910 \mathrm{~km})$ travelled. $(I Q R=$ interquartile range; $\min .=$ minimum; $\max .=$ maximum.)

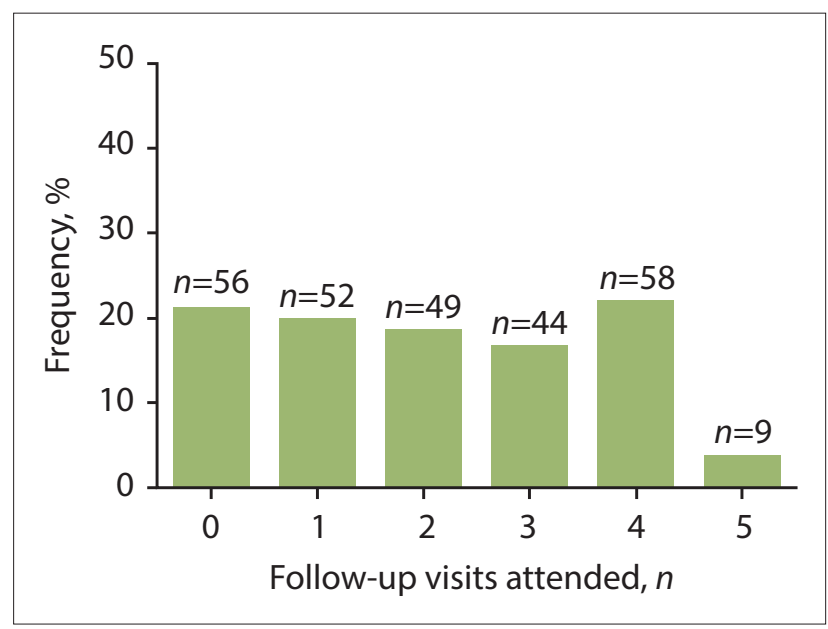

Fig. 4. Frequencies (\%) of the number of follow-up visits that participants $(\mathrm{N}=268)$ attended. Patients were divided into six groups, based on their follow-up status: those who attended none of the five follow-up visits were grouped into group $0(\mathrm{n}=56)$, those who attended all follow-up visits were grouped into group $5(\mathrm{n}=9)$, and those who attended only a subset of scheduled follow-up visits were grouped into groups 1 - 4. The total number of participants in each follow-up group is indicated.

Table 1. Univariate analysis of predictors influencing the number of follow-up visits attended by patients

\begin{tabular}{lll}
\hline \multicolumn{3}{l}{ number of follow-up visits attended by patients } \\
\hline Age & IRR $(\mathbf{9 5 \%}$ CI $)$ & p-value \\
Sex & $1.008(1.002-1.014)$ & $0.012^{*}$ \\
Travel distance & $0.944(0.798-1.117)$ & 0.501 \\
BMI & $0.999(0.999-0.999)$ & $0.024^{*}$ \\
Smoking & $0.982(0.969-0.995)$ & $0.008^{*}$ \\
Alcohol use & $1.085(0.914-1.289)$ & 0.35 \\
Comorbid conditions & $1.036(0.868-1.238)$ & 0.695 \\
Days to admission & $0.902(0.762-1.067)$ & 0.227 \\
Days to surgery & $1.003(0.992-1.014)$ & 0.266 \\
HIV status & $0.864(0.745-1.002)$ & $0.053^{*}$ \\
IRR $=$ incidence rate ratio; CI $=$ confidence interval; BMI = body mass index. & \\
*Significant at $p<0.05$ level. &
\end{tabular}


A Poisson multivariate regression was performed to evaluate factors that were independently associated with the number of follow-up visits attended. The model included age, travel distance, BMI and HIV status. Age did not contribute to the model, however, and was subsequently excluded from the final model. The final model $(p<0.001)$ showed that the included risk factors were significantly associated with the number of follow-up visits attended: more specifically travel distance (incidence rate ratio (IRR) $0.999,95 \%$ CI 0.999 - 1.000; $p=0.030$ ), BMI (IRR 0.980, 95\% CI 0.966 - 0.994; $p=0.004$ ) and HIV status (IRR $0.841,95 \%$ CI $0.725-0.975 ; p=0.022$ ).

\section{Discussion}

The objective of this study was to describe potential factors that contribute to loss to follow-up as seen in orthopaedic patients attending the sole public orthopaedic service provider in the Northern Cape, Kimberley Provincial Hospital.

The first main finding of this study was that travel distance, BMI and HIV status were the most important predictors of attendance for follow-up visits, with an increased travel distance, increased BMI and HIV-positive status making it more likely for patients not to return for follow-up. Alcohol use, smoking and male gender have been reported as major risk factors for loss to follow-up in the literature, ${ }^{[4,5,8]}$ but this was not found in the present study. Zelle et al ${ }^{[4]}$ reported travel distance not to be a predictor of loss to follow-up, ${ }^{[4]}$ while the present study highlights this to be a risk factor. A possible explanation could be a difference in socioeconomic status, which is not reported in either the present study or that of Zelle et al. ${ }^{[4]}$ This is therefore a potential avenue for future work.

The second main finding of this study was that despite proactive scheduling for appointments, providing study patients with a separate follow-up room to reduce waiting time and remuneration for travel expenses, $20.9 \%$ of patients did not attend a single follow-up visit and only $33.2 \%(n=89)$ attended follow-up visits at 6 months or 1 year (data not shown). Clinically, it is important to see patients for at least 6 months after any surgery to evaluate fracture healing. It is therefore very concerning that, even under special circumstances not normally present in healthcare settings, we failed to successfully follow up on such a large proportion of patients.

In the present study, increased BMI was associated with fewer follow-up visits attended by patients. We also found that for every unit increase of BMI, there was a $10 \%$ decrease in the follow-up rate. This could potentially be explained by difficulty ambulating long distances with crutches to arrive at public transport locations, and then travelling vast distances in crowded vehicles with little space for walking aids. In contrast, it is tempting to speculate that, similar to smokers, ${ }^{[2]}$ individuals with higher BMIs are not as motivated to change their behaviour for health-related purposes.

Patients who do not experience any postoperative complications may potentially not be likely to travel long distances and spend time away from work just to confirm their personal perception that their treatment was successful. In support of this, Casp et al. ${ }^{[3]}$ reported that patients who reported feeling well did not see the need to attend follow-up visits. They concluded that the lack of follow-up results from a voluntary decision by the patient not to seek further medical care, rather than the failure of logistics or scheduling to provide the patient with adequate care and followup appointments. In the present study, patients were contacted telephonically when they failed to report for follow-up visits and several patients did indeed report good recovery and therefore did not feel the necessity to attend. Additional anecdotal reports included experiencing difficulty with public hospital transport, which is often overcrowded, and not being able to afford to travel to the hospital privately.

In our final multivariate model, travel distance, BMI and HIV status were predictors of the number of follow-up visits attended. However, none of these associations were very strong, which highlights the complexity of the phenomenon of loss to follow-up. It is clear that there are several factors that influence a patient's decision and ability to attend follow-up visits, globally and in SA. In the present study, only ten possible variables were described, and patient education, family status and other socioeconomic status factors that are potential important predictors were not recorded.

The rural areas and metropoles in SA are divided into specific health service referral areas for each public healthcare institution. This precludes an individual from seeking medical help at a health institution outside his or her drainage area, with Kimberley Provincial Hospital being the only institution delivering a tertiary surgical service in the Northern Cape. Patients will not be serviced if they do not live in their respective drainage areas and therefore cannot go to a different health institution if they have a problem following surgery. This therefore excludes loss to follow-up as a result of dissatisfaction with the service provider. In the present study, we attempted to use the waiting time between admissions to surgery as a proxy for patient satisfaction, with the rationale being that patients who have to wait very long from admission to surgery may be more likely to not return for follow-up visits. We failed to report an association, however, and future research in a public setting should attempt to measure patient satisfaction more accurately.

An additional factor to consider is the ability to contact patients, even when contact details are provided. The availability of mobile phones creates the impression that all patients will be able to be contacted by means of a phone call, but this is not always the case. SA's mobile phone services are considered among the most expensive in the world. The fact that a cellphone starter pack is accessible at a cheaper rate than reloading data or airtime using an existing phone number causes problems with follow-up, since patients often change phone numbers, generally without informing medical service providers, and therefore cannot be contacted.

Obtaining extra funding to conduct trials in limited-resource environments with vast travel distances, so that patients can be remunerated to attend follow-up appointments, could potentially alleviate the problem of loss to follow-up. It is, however, important to keep in mind that travel distance is only one of various factors associated with limited follow-up, and that even in our model there are likely to be several additional risk factors that this study did not measure or report on. Nevertheless, this study does highlight the complexities of circumstances surrounding patients who are lost to follow-up.

\section{Conclusions}

Factors that contribute to loss to follow-up in an SA setting are similar to those reported in the literature, but another layer of complexity is added in the public setting, where increased travel distance and time for patients to attend hospital visits are involved. A multicentre approach could potentially provide an improved strategy in protocol planning to accommodate these factors and improve our patient follow-up and related research quality.

\section{Declaration. None.}

Acknowledgements. We thank Mr Michael McCaul of the Division of Epidemiology and Biostatistics, Stellenbosch University, for his help in data analysis. 
Author contributions. DHSB: contributed towards the conception and design of the research, acquisition of data, drafting and reading of the manuscript; CAvdW: contributed towards conception and design of the protocol and supervision of surgery of the study population; EB: contributed towards acquisition of data; MCB: contributed towards the conception and design of the research, drafting and reading of the manuscript; NF: contributed towards the conception and design of the research, drafting and reading of the manuscript.

Funding. Funding for patient reimbursement was received from the South African Orthopaedic Association.

Conflicts of interest. None.

1. Elsoe R, Ostgaard SE, Larsen P. Population-based epidemiology of 9767 ankle fractures. Foot Ankle Surg 2018:24(1):34-39. https://doi.org/10.1016/j.fas.2016.11.002

2. Neuner B, Fleming M, Born R, et al. Predictors of loss to follow-up in young patients with minor trauma after screening and written intervention for alcohol in an urban emergency department. J Stud Alcohol Drugs 2007;68(1):133-140. https://doi.org/10.15288/jsad.2007.68.133

3. Casp AJ, Wells J, Holzgrefe R, Weiss D, Kahler D, Yarboro SR. Evaluation of orthopedic trauma surgery follow-up and impact of a routine callback program. Orthopedics [Internet] 2017;40(2):e312-e316. https://doi.org/10.3928/01477447-20161229-01
4. Zelle BA, Buttacavoli FA, Shroff JB, Stirton JB. Loss of follow-up in orthopaedic trauma: Who is getting lost to follow-up? J Orthop Trauma 2015;29(11):510-515. https://doi.org/10.1097/BOT 0000000000000346

5. Madden K, Scott T, McKay P, et al. Predicting and preventing loss to follow-up of adult trauma patients in randomized controlled trials. J Bone Jt Surg Am 2017;99(13):1086-1092. https://doi.org/10.2106 JBJS. 16.00900

6. Tejwani NC, Takemoto RC, Nayak G, Pahk B, Egol KA. Who is lost to followup? A study of patients with distal radius fractures. Clin Orthop Relat Res 2010;468(2):599-604. https://doi.org/10.1007/ s1 1999-009-0968-6

7. Mclean RR, Hannan MT, Epstein BE, et al. Elderly cohort study subjects unable to return for follow-up have lower bone mass than those who can return. Am J Epidemiol 2018;151(7):689-692. https://doi. org/10.1093/oxfordjournals.aje.a010263

8. Murnaghan ML, Buckley RE. Lost but not forgotten: Patients lost to follow- up in a trauma database. Can J Surg 2002;45(3):191-195.

9. Murray D, Britton A, Bulstrode C. Loss to follow-up matters. J Bone Jt Surg Br 1997;79(2):254-257 https://doi.org/10.1302/0301-620X.79B2.0790254

10. Daffner SD, Hilibrand AS, Riew KD, Virginia W. Why are spine surgery patients lost to follow-up? Global Spine J 2013;3(1):15-20. https://doi.org/10.1055/s-0033-1337120

11. Statistics South Africa. Provincial profile: Northern Cape. Census 2011. http://www.statssa.gov.za/ publications/Report-03-01-72/Report-03-01-722011.pdf (accessed 11 October 2018).

Accepted 24 May 2018 